Betts Kim (Orcid ID: 0000-0001-5855-1911)

Kisely Steve (Orcid ID: 0000-0003-4021-2924)

Alati Rosa (Orcid ID: 0000-0002-9240-3450)

\title{
Prenatal cannabis use disorders and offspring primary and secondary educational outcomes
}

Running head: Prenatal cannabis and educational outcomes

Abstract word count is 312 words.

Main text word count is 2,410 words.

There are 4 tables and 0 figures.

Dr Kim S. Betts (corresponding author), PhD, MBiostats, MPH.

School of Public Health,

Building 400, Kent Street, Bentley,

Curtin University,

WA 6101, Australia

kim.betts@curtin.edu.au

Prof Steve Kisely MD, PhD, DMedRes

School of Medicine, University of Queensland, Brisbane, Australia

s.kisely@uq.edu.au

Prof Rosa Alati, PhD, MApplSc(Health Sc), BA(Hons).

School of Public Health, Building 400, Kent Street Bentley, Curtin University, WA 6101 Email: rosa.alati@curtin.edu.auHonorary Professor, Institute for Social Science Research, University of Queensland, QLD

Acknowledgements: We thank the Centre for Health Record Linkage (CHeReL) within the NSW Department of Health for making this project possible.

This article has been accepted for publication and undergone full peer review but has not been through the copyediting, typesetting, pagination and proofreading process which may lead to differences between this version and the Version of Record. Please cite this article as doi: $10.1111 /$ add. 15629

This article is protected by copyright. All rights reserved. 
Ethics: Ethics approval was granted from Cancer Institute NSW, NSW Population and Health Service Research Ethics Committee (HREC/18/CIPHS/22) and Curtin University.

Access to data: $\mathrm{KB}$ had access to the complete dataset used in the study and takes responsibility for the integrity of the data and accuracy of the data analyses.

Declaration of interest: All authors declare no conflicts of interest

Funding/Support: None.

Financial relationships: There are no financial relationships to report.

\section{Contributors Statement Page}

Dr Kim Betts has been designated principal author and was responsible for the bulk of the literature review, drafting, statistical analysis and discussion. RA and SK played a major role in data acquisition and drafting. All authors approved the final version and are accountable for the all aspects of the work in ensuring that questions related to the accuracy or integrity of any part of the work are appropriately investigated and resolved.

Key words: Prenatal cannabis use, offspring educational outcomes, longitudinal data, health administrative data.

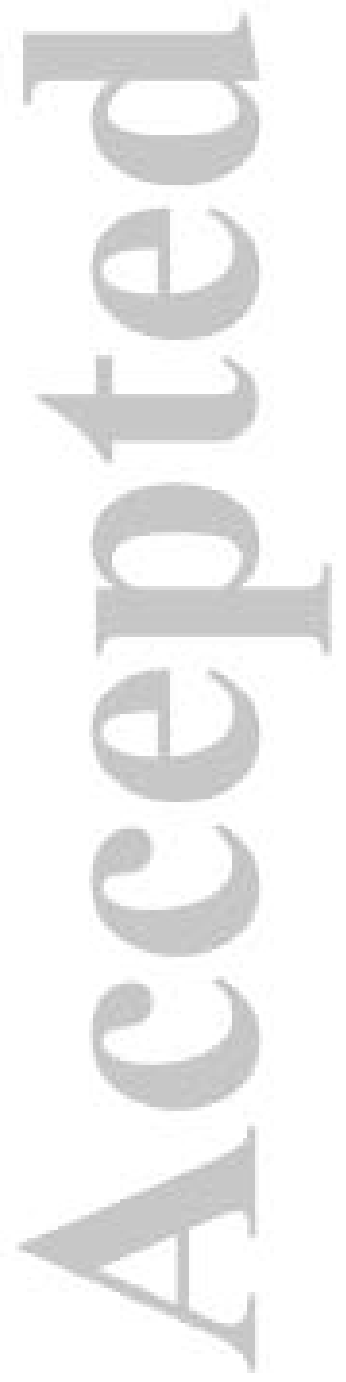




\begin{abstract}
Background and Aims: Cannabis use is increasing among women of reproductive age, warranting a greater understanding of the impact of prenatal cannabis use on offspring developmental outcomes. We tested for a potential relationship between prenatal cannabis use disorders (CUD) and offspring educational outcomes across primary and secondary school.
\end{abstract}

Design: Data were drawn from the New South Wales (NSW) Perinatal Data Collection, which included all live births in the Australian state of NSW between January 2003 and December 2005. These were linked with the NSW Admitted Patient Data collection for mothers and offspring, and the NSW National Assessment Program - Literacy and Numeracy (NAPLAN).

Setting: New South Wales, Australia.

Participants: A total of 189,558 offspring who completed the NAPLAN in grades 3, 5 and 7 (resulting in 568,674 examination periods).

Measurements: The exposure variable was ICD-10 Cannabis Use Disorders (CUD: F13.0F13.9). The study included five outcome variables measured at three time points, as not meeting the minimum national standards for: (i) numeracy, (ii) reading, (iii) spelling, (iv) writing, and (v) grammar and punctuation.

Findings: In unadjusted analyses, prenatal CUD was associated with an increased risk for not meeting the national minimum standard of all outcomes [odds ratios (OR) ranging from 3.42 (95\% confidence interval $(\mathrm{CI})=2.94,3.99)$ to $4.17(95 \% \mathrm{CI}=3.55,4.91)]$, with no evidence for an interaction across time. However, the associations attenuated greatly after exact matching by covariates, with reading and numeracy no longer associated with prenatal CUD, while the increased risk of the other outcomes ranged $\mathrm{OR}=1.31(95 \% \mathrm{CI}=1.09,1.57)$ to $\mathrm{OR}=1.40(95 \%$ $\mathrm{CI}=1.17,1.68)$.

Conclusion: Socioeconomic status appears to confound the association between prenatal cannabis use disorder and poor educational performance in offspring. 


\section{Introduction}

The last decade has seen a liberalisation of attitudes to cannabis use in developed countries and increased availability and use among adults (1). One group of particular concern are women of reproductive age, with recent data indicating that self-reported recreational cannabis use among pregnant mothers in the USA doubled between 2002 and 2017 (2). The risks associated with recreational use of cannabis during pregnancy have not been fully established. High-quality cohort studies conducted in a number of settings indicate that prenatal cannabis use increases the risk of low birth weight and premature birth $(3,4)$. Much less is known about the possible behavioural and psychological outcomes of exposure to cannabis during pregnancy. The limited evidence on the impact of prenatal cannabis use on neurodevelopmental outcomes in offspring comes from three prospective cohort studies (58). Although little evidence of altered neonatal or infant behaviours or abilities arising from prenatal cannabis use have been found, increased behaviour problems were reported in later childhood, including hyperactivity and impulsivity, as well as altered cognitive development, particularly on tasks measuring executive functioning (9). These observations have been supported by animal studies, which found a range of cognitive deficits in rats exposed to cannabinoid agonists during pregnancy, including disrupted memory retention (10), impaired social discrimination (11) and object recognition (12). These deficits were correlated with brain structural and functional changes (13).

An important consideration in epidemiological studies of prenatal cannabis use is the ability to account for socio-economic and other important confounding factors. There are strong relationships between social disadvantage, cannabis use and academic achievement which are well established $(14,15)$. Those who use cannabis are also much more likely to use other drugs $(16,17)$, which makes it difficult to accurately assess the role of prenatal cannabis use, 
as opposed to that of other drugs, in the association with offspring's development. Further, substance use disorders co-occur with mental health disorders $(18,19)$, which can further confound the relationship between prenatal exposure to cannabis use and offspring's neurodevelopmental outcomes. Previous research in this field has been unable to adequately adjust for co-occurring substance use disorders and mental health disorders (i.e., depression, anxiety), either due to lack of information or the statistical power necessary to model a potentially large number of confounders. Given the evidence above, we expect that any relationship between prenatal cannabis use and poor educational outcomes will be partly explained by lower socio-economic status.

In this study, we linked the standardised educational examination results of 189,558 children from New South Wales (NSW), each having undergone standardised academic testing at three time points (grades 3,5 and 7) with maternal perinatal and inpatient data collections during pregnancy. This resulted in more than half a million observations $(n=568,674)$ with which to examine the association between prenatal DSM-IV Cannabis Use Disorders (CUD) and five domains of educational outcomes taken from the Australia's National Assessment Program - Literacy and Numeracy (NAPLAN). Importantly, the large sample size and comprehensive nature of the perinatal and inpatient data collections allowed us to adjust for the other mental and substance use disorders, in addition to socioeconomic factors, by using an exact matching approach. In Australia, cannabis has long been the most widely used illicit drug, with a population-based longitudinal study carried among Australian youth in the 1990's showed one in five adolescents reported using cannabis in their mid-teens, with $9 \%$ of females reporting daily use (20).

Lastly, as the same five educational domains were tested at three time points, we were also able to observe how the relationships change over time from early primary school through to 
early high school. Two recent reviews concerning the neurodevelopmental impacts of prenatal cannabis exposure have found that associations with a range of measures, including cognitive ability, behaviour problems and motor skills appear to strengthen as the child ages $(9,13)$. The reasons for this are unknown, although it has been suggested that the effects of prenatal cannabis exposure become more apparent as brain development matures (9). In this study we tested the association between prenatal CUD and educational outcomes among a large population sample, and examined if the association changed with age.

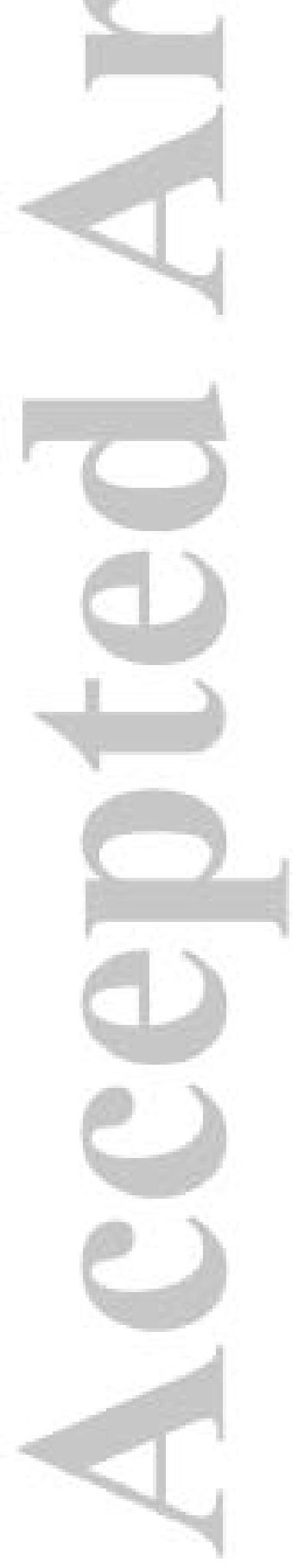




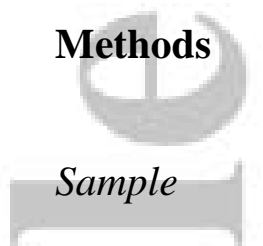

The sample included all mother-offspring pairs from inpatient live births in the Australian state of New South Wales (NSW) between January 2003 and December 2005 where children subsequently completed NAPLAN in grades 3, 5 and 7 ( $n=189,558$ offspring; resulting in 568,674 examination periods). Mothers and offspring were linked across the following three datasets: the NSW perinatal data collection (PDC); the NSW admitted patient data collection (APDC) (both of which include all public and private hospital patients), and; the NSW NAPLAN data collection. For all mothers, PDC and APDC data were available for the duration of their pregnancy ( $\sim 9$ months before the birth). The data collections are linked by the NSW Centre for Health Record Linkage (CHeReL), with further information on the probabilistic linkage and quality assurance methods described on their webpage (21). Ethics approval was granted from Cancer Institute NSW, NSW Population and Health Service Research Ethics Committee (HREC/18/CIPHS/22) and Curtin University.

\section{The outcome variable}

The NAPLAN is an annual education examination undertaken by all governmental and nongovernmental school students in grades 3, 5, 7 and 9 in Australia. Schools in all states and territories, including NSW, administered NAPLAN every May from 2008 to 2019. The exams are delivered in the English language and assess five domains, including reading, spelling, grammar, writing and numeracy. In addition to bands which rank students by level of achievement, students are judged against the National Minimum Standards (meeting/ not meeting). Students who score below the standard have not achieved the learning outcomes expected for their year level and are at risk of being unable to progress satisfactorily at school without targeted intervention (22). Students classified as achieving less than the national 
minimum standard ranged from $2.6 \%(n=14,644)$ for numeracy to $4.3 \%(n=25,973)$ for writing. Completion rates are very high. In the most recent year of administration, these exceeded $95 \%$ in NSW over the five domains and across the relevant grades for this study (3, 5 and 7). In this study, the outcome variable was not having met the National Minimum Standard on the five domains as the outcome variables.

\section{Cannabis use disorders and comorbid disorders}

All data regarding maternal substance use and mental health disorders during pregnancy came from the APDC and included all principal or secondary diagnoses. Cannabis use disorders were defined according to ICD-10 criteria for mental and behavioural disorders due to use of cannabinoids [F12.0-F12.9]. Comorbid substance use disorders were similarly defined for other substance categories in ICD-10 [alcohol, F10.0-F10.9; opioids, F11.0F11.9; sedative/hypnotics, F13.0-F13.9; cocaine, F14.0-F14.9; other stimulants, F15.0-F15.9; hallucinogens, F16.0-F16.9; tobacco, F17.0-F17.9; volatile solvents, F18.0-F18.9; multiple drug use, F19.0-F19.9]. Mental health disorders were also extracted, including non-affective psychotic disorders [F20.0-F29.0], bipolar depressive disorders [F30.0-F31.9], unipolar depressive disorders [F32.0-F39.9] and anxiety disorders [F40.0-48.9]. To be defined as occurring during the prenatal period, the episode of care within which the diagnosis was noted must fall between the date of conception and date of delivery (conception date was estimated as the delivery date minus the gestational age at delivery).

\section{Potential confounders}

A number of a priori confounders were included in the models, which could explain the relationship between the exposure and outcomes. These were extracted from the PDC and NAPLAN, and includes Socio-economic Indexes for Areas (SEIFA codes) used by the Australian Bureau of Statistics to rate postcodes by relative economic disadvantage and 
advantage (quartiles), type of school (Government, Catholic, independent/private), student having a language background other than English (LBOTE; yes/no), maternal age at the birth

$(<18,18-25,26-30,31-35,>35)$, number of previous pregnancies $(0,1,2+)$, prenatal smoking status (yes/no), gestational diabetes, pre-existing diabetes, gestational hypertension and preexisting hypertension, delivery method, resuscitation and need for intensive care. Birth weight and gestational age were not included as confounders because previous research shows they are a result of prenatal CUD $(3,4)$, and therefore may lie on the causal pathway.

Statistical analyses

Univariable relationships between cannabis use disorders (CUD) with the potential confounders were firstly undertaken, using appropriate statistical tests [proportions with Chisquare test for categorical confounders and means with t-tests for continuous confounders].

Next, we then tested the associations between CUD with each of the NAPLAN tests in the original sample using marginal models with an unstructured covariance matrix to account for non-independence among repeated measures, using the R package GEE (23). Subsequently, we used an exact matching algorithm available in the R package MatchIt (24) to match exposed subject to unexposed subjects with exactly the same values on all covariates. Exposed and unexposed subjects which are unable to be matched are removed, while unexposed subjects are given a weight to account for the variable size of subgroups (i.e., groups of exposed/unexposed subjects with the same covariate values). The matched data was then modelled using the R package survey (25), which accounts for the weighting and non-independence among repeated measures. Lastly, it is important to note that the analyses and results of this study were exploratory, and not the result of a pre-registered analysis plan.

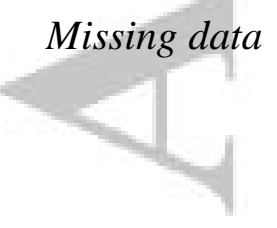

This article is protected by copyright. All rights reserved. 
In our data, all participants were of the appropriate age to have completed the first 3

NAPLAN assessments. To begin with, we had a total of 673,720 student records for these

assessments (i.e., each student could have one record for each of three assessment grades, if they were enrolled at a NSW school during the test). From this initial number, 36,822 (5.5\%) records did not have an assessment grade for reasons unknown (absent or excluded due to a disability or learning problem). From the remaining 636,898 assessment result records, $69,478(11 \%)$ were removed because the student was missing data for at least one of the three assessments.

When comparing the outcomes between the 69,478 who were removed and the 567,420 who remained in the analysis, we found a much higher proportion of those removed were classed as not meeting the national minimum standards [Reading: Missing $=10.5 \%$, not missing $=3.5 \%, \mathrm{p}<0.001$; Spelling: Missing $=11.8 \%$, not missing $=3.6 \%, \mathrm{p}<0.001$; Grammar: Missing $=11.9 \%$, not missing $=4.3 \%, P, 0.001$; Writing: Missing $=13.5 \%$, not $\operatorname{missing}=4.6 \%$, $\mathrm{p}<0.001$; Numeracy: Missing=8.5\%, not missing $=2.6 \%, \mathrm{p}<0.001]$. This is expected, as many of these children missing NAPLAN scores may have sat one or two NAPLAN's at some stage of their schooling, but may have been excluded at another assessment due to a disability or learning/behaviour difficulty. These students would be expected to be more likely to not meet the national minimum standards. Unfortunately, we are not given information regarding why a student did not sit the assessments. 


\section{Results}

The prevalence of prenatal CUD in our sample was $0.47 \%$ (887 out of 187011 pregnancies).

Offspring exposed to CUD were more likely to not meet the minimum national standards for reading $(10.9 \%$ versus $3.4 \%$, $\mathrm{p}<0.001)$, spelling $(13.2 \%$ versus $3.5 \%, \mathrm{p}<0.001)$, grammar

( $14.2 \%$ versus $4.2 \%, \mathrm{p}<0.001)$, writing $(16.3 \%$ versus $4.5 \%, \mathrm{p}<0.001)$, and numeracy $(8.4 \%$ versus $2.6 \%, \mathrm{p}<0.001)$. Table 1 shows the univariable associations between the potential confounders with maternal prenatal cannabis disorder (CUD). Unsurprisingly, rates of prenatal smoking were much higher among the CUD group, with $81.7 \%$ of these mothers smoking compared with only $12.2 \%$ among the women without CUD $(\mathrm{p}<0.001)$. In addition, all other substance use and mental health disorders occurred far more frequently among the CUD group [e.g., depressive disorders, $10.3 \%$ vs $0.8 \%$ ( $<<0.001)$; opioid use disorders, $10.0 \%$ vs $0.3 \%(\mathrm{p}<0.001)$; alcohol use disorders, $5.5 \%$ vs $0.1 \%(\mathrm{p}<0.001)$; and psychotic disorders, $3.8 \%$ vs $0.1 \%(\mathrm{p}<0.001)$ ]. Women with CUD were less likely to have gestational or pre-existing diabetes or hypertension, were less likely to have a caesarean delivery or a baby requiring resuscitation, although were more likely to have a baby required observation [33.6\% vs $14.0 \%(\mathrm{p}<0.001)]$ or NICU admission [7.6\% vs $2.4 \%(\mathrm{p}<0.001)]$. We observed a clear social gradient, whereby women with CUD were far more likely to come from lower SES backgrounds and more likely to send their child to a public (tax-payer funded) school. In addition, $50 \%$ of mothers with CUD were below the age of 25 compared with only $20 \%$ below age 25 among those without CUD, while mothers with CUD had more previous pregnancies than those without CUD.

From the initial 891 mothers who had CUD in pregnancy, 593 (67\%) were exactly matched to at least one control. The additional 298 Women with CUD who had no exact matches, along with control subjects who had no matches with Women with CUD, were excluded from 
the matched data. This resulted in a matched sample of 30,717 , which comprised only $16 \%$ of the initial sample, reflecting the fact that the majority of mothers who did not have CUD differed in many respects to mothers who did and reinforcing the importance of rigorous adjustment.

Table 2 shows the associations between prenatal CUD and the NAPLAN examination results in the full sample (unmatched) and the matched sample. Prior to matching, children exposed to prenatal CUD were at a consistently greater risk of not meeting the national minimum standards across all domains, with odds ratios ranging between 3.4 and 4.2. Further, there was no evidence for an interaction, meaning the impact of prenatal CUD on exam results did not differ by the grade. In the matched sample, the effect estimates attenuated markedly, with associations with the reading and numeracy exam results attenuating to the null.

Lastly, the attenuation after matching was far greater than we had expected. In response, we undertook two ad-hoc analyses, in which unadjusted and matched associations were estimated between Alcohol Use Disorders (AUD) and Opioid Use Disorders (OUD) with each of the outcomes. This was done to see whether the attenuation after matching was specific to CUD, or if other substance use disorders known to impact offspring health and development similarly attenuated. The results, which are shown in table 3 , showed that these disorders also attenuated markedly, with OUD exhibiting similar, though slightly stronger, associations to CUD before and after matching. In addition, AUD had some of the strongest unadjusted associations, but after matching only the association with numeracy remained. 


\section{Discussion}

We undertook the largest and most comprehensive study to date concerning the association between prenatal cannabis use disorder and offspring educational outcomes, spanning five scholastic domains tested three times across five years of schooling on a population sample with very high completion rates. After applying a sophisticated method to adjust for the confounding introduced by additional substance use and mental health disorders, we found only a weak association between prenatal CUD and an increased risk of failure to meet the national minimum standard in spelling, grammar and writing, and no evidence of an association with reading and numeracy. Further, we found no interaction with time, indicating no empirical support that the impact of prenatal CUD on offspring outcomes became stronger or weaker with offspring age.

Our study clearly demonstrates that much of the observed association between prenatal CUD and offspring education performance is dependent on comorbidities and socioeconomic factors. The attenuation of the effect estimates following exact matching was far greater than we had anticipated, not only for prenatal CUD but also for prenatal AUD and OUD (alcohol and opioid use disorders). Taken together, this suggested that much of the association between prenatal substance use disorders in general, including CUD, and offspring scholastic outcomes was due to exposed children being much more likely to attend public schools, come from lower socioeconomic areas, and to be born to younger mothers, all strong indicators of low socio-economic status and social disadvantage.

Though our findings point to a socio-economic rather than an intra-uterine effect, our study should not be seen as evidence for the innocuous nature of prenatal cannabis use. We suggest similar recommendations made for findings between prenatal alcohol use and offspring outcomes: that without an empirically proven safe level of exposure, abstinence is the only 
reasonable advice the medical community can provide to pregnant mothers $(26,27)$. Further, we only investigated relationship between cannabis use disorders and offspring' educational performance. Hence these findings should be seen as only applying to this domain of child development. More research is needed assessing the associations between prenatal cannabis use with a broader range of behavioural and social outcomes among offspring.

Despite observing marked attenuation upon adjustment for socioeconomic factors, the weak associations remaining warrant consideration of a biological mechanism. Animal studies have estimated that one-third of plasma THC crosses the fetoplacental barrier, with exposure associated with changes to key gene expressions and alterations in important neurological systems in rats (9). Alterations in the striatal encephalin/D2 receptors and the opioid system in the amygdala were observed in aborted human foetuses exposed to maternal cannabis (28), suggesting that exposure could alter foetal brain development trajectory. In our study, we also identified specific effects, such that prenatal cannabis exposure was associated with spelling, grammar and writing, but not reading or numeracy. Previous studies indicate that exposure is linked with specific cognitive deficits, such as impairment in abstract and visual reasoning, in addition to visuoperceptual processing (9). However, considering the level of attenuation post-adjustment, and small effect estimates, we would hesitate to draw conclusions regarding specific effects in our own data.

In addition to the many strengths of our study outlined above, our data includes a number of important limitations. Firstly, many mothers with prenatal CUD are likely to continue using cannabis and other substances after their pregnancy, which may influence both their parenting abilities and their offspring's exploration of substance use. Both of these factors, which we did not have any information regarding, are likely to also negatively affect offspring scholastic achievement. In fact, the weak associations we found between prenatal 
CUD and offspring education are likely within the bounds of residual confounding. In other words, the associations may be explained by the many lifestyle and behavioural factors associated with cannabis use that were not included in our data. Further, as CUD has been found to have a genetic pre-disposition, it is possible that early experimentation of cannabis use among exposed offspring accounted for the association.

Secondly, our exposure was CUD, and not cannabis consumption. Our data included no information regarding how much cannabis mothers consumed, nor at what point, intervention for CUD was implemented and how effective it was at preventing or reducing further cannabis consumption. This means our exposure will likely have varied widely concerning how much cannabis was actually consumed during pregnancy and at what point in pregnancy. A recent retrospective study found that cannabis exposure prior, but not after, maternal knowledge of pregnancy, was not associated with offspring neurodevelopmental outcomes, while exposure after maternal knowledge was (29). This suggests the failure to stop consuming cannabis in early pregnancy is crucial to child outcomes.

Although we don't know the timing of cannabis exposure, the prevalence of CUD in our sample was only $0.5 \%$ compared with the $3.2 \%$ of pregnant mothers in the USA who reported past month cannabis youth in 2002-2003 (2). Thus, by using CUD as an exposure we likely included only the heaviest cannabis consumers among our exposed group. Importantly, this also makes it possible that out effect estimates were downwards biased, as the control group is likely to have included mothers who used cannabis but did not have a clinical disorder. This downward bias may have been further exacerbated by the exact matching, as CUD is associated with other disorders. Hence, many of the matches who had these other disorders but not CUD, may have still used cannabis. Further, the same limitation can be extended to the other outcomes, AUD and OUD, for which alcohol use may be of 
particular concern, considering that non-disordered alcohol use is common. We encourage future studies based on self-reported consumption data to replicate our findings on educational outcomes.

Thirdly, only a small proportion of students (between $2.6 \%$ to $4.3 \%$ ) did not meet the national minimum standards for the given assessment. Thus, our outcome represented quite an extreme measure of poor educational performance. It is possible that had we used a less stringent cut-off, we might have had more variability in the outcome with which to observe more subtle differences. On the other hand, our data excluded the lowest performing students who were excluded from the NAPLAN. Thus, the relationship we found between prenatal CUD and educational outcomes may not generalise to children with severe learning or behavioural disabilities, and this will need to be explored is studies with data on such children.

Lastly, we must consider that the confounding effect of socioeconomic status may partly result from selection bias, as people from disadvantaged backgrounds may be more likely to be given a diagnosis due to greater involvement with the justice system. If this was the case, then the effect of socioeconomic status may be larger in this sample than samples using selfreported measures, thereby wrongly attenuating the association of interest. However, recent studies have found a higher incidence of substance use among pregnant mothers from lower socioeconomic backgrounds and among Aboriginals. In addition, court diversion schemes usually mandate state-run outpatient treatment whereas our data concern inpatient data from both the public and private system $(30,31)$. Therefore, this is unlikely to be the case in our study.

In summary, our large population-based study indicated that prenatal cannabis use disorders are at best weakly associated with offspring poor educational performance in childhood and 
early adolescence. In the face of increasing cannabis consumption, including among pregnant women, more research is needed to assess the risks posed to the developing offspring.

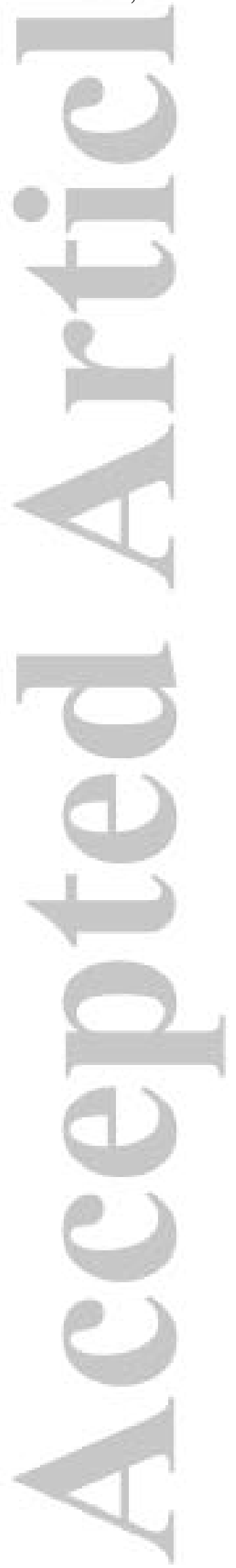

This article is protected by copyright. All rights reserved. 
Table 1: Distribution of potential confounders by maternal prenatal cannabis disorder

\begin{tabular}{|c|c|c|c|c|c|c|}
\hline \multirow{2}{*}{$\begin{array}{l}\text { Predictor } \\
\text { Categorical variables }\end{array}$} & \multicolumn{2}{|c|}{ No cannabis use $\mathrm{dx}$} & \multicolumn{2}{|c|}{ Cannabis use $\mathrm{dx}$} & \multirow[t]{2}{*}{ chi square } & \multirow[t]{2}{*}{$p$ value } \\
\hline & $\%$ & $\mathbf{n}$ & $\%$ & $\mathbf{n}$ & & \\
\hline \multirow[t]{2}{*}{ Prenatal smoking (no) } & 87.8 & 165342 & 18.3 & 163 & 3906.8 & $<0.001$ \\
\hline & 12.2 & 22955 & 81.7 & 728 & & \\
\hline \multirow{2}{*}{ Prenatal diabetes (no) } & 99.5 & 187255 & 99.7 & 888 & 0.4 & 0.520 \\
\hline & 0.6 & 1042 & 0.3 & 3 & & \\
\hline \multirow[t]{2}{*}{ Pre-existing diabetes (no) } & 95.4 & 179544 & 98.3 & 876 & 17.0 & $<0.001$ \\
\hline & 4.7 & 8753 & 1.7 & 15 & & \\
\hline \multirow{2}{*}{ Prenatal hypertension (no) } & 98.9 & 186288 & 99.9 & 890 & 6.8 & 0.009 \\
\hline & 1.1 & 2009 & 0.1 & 1 & & \\
\hline \multirow[t]{2}{*}{ Pre-existing hypertension (no) } & 94.5 & 177919 & 96.3 & 858 & 5.2 & 0.022 \\
\hline & 5.5 & 10378 & 3.7 & 33 & & \\
\hline \multirow{2}{*}{ Induction (no) } & 83.8 & 157808 & 91.8 & 818 & 41.3 & $<0.001$ \\
\hline & 16.2 & 30489 & 8.2 & 73 & & \\
\hline \multirow{2}{*}{ Breach (no) } & 95.4 & 179707 & 95.2 & 848 & 0.1 & 0.767 \\
\hline & 4.6 & 8590 & 4.8 & 43 & & \\
\hline \multirow{2}{*}{ Caesar (no) } & 71.9 & 135322 & 82.5 & 735 & 49.1 & 0.000 \\
\hline & 28.1 & 52975 & 17.5 & 156 & & \\
\hline \multirow{2}{*}{ Caesar due to fetal distress (no) } & 96.6 & 181911 & 95.5 & 851 & 2.9 & 0.087 \\
\hline & 3.4 & 6386 & 4.5 & 40 & & \\
\hline \multirow{2}{*}{ Resuscitation (no) } & 53.5 & 100809 & 59.3 & 528 & 11.4 & 0.001 \\
\hline & 46.5 & 87488 & 40.7 & 363 & & \\
\hline \multirow[t]{2}{*}{ Admitted to nursey (observation) (no) } & 86.0 & 161888 & 66.4 & 592 & 279.1 & $<0.001$ \\
\hline & 14.0 & 26330 & 33.6 & 299 & & \\
\hline \multirow{2}{*}{ Admitted to NICU (no) } & 97.6 & 183823 & 92.4 & 823 & 103.2 & $<0.001$ \\
\hline & 2.4 & 4453 & 7.6 & 68 & & \\
\hline \multirow[t]{2}{*}{ Prenatal psychotic disorder (no) } & 99.9 & 188139 & 96.2 & 857 & 1181.8 & $<0.001$ \\
\hline & 0.1 & 158 & 3.8 & 34 & & \\
\hline \multirow{2}{*}{ Prenatal anxiety disorder (no) } & 99.4 & 187234 & 94.6 & 843 & 345.1 & $<0.001$ \\
\hline & 0.6 & 1063 & 5.4 & 48 & & \\
\hline Prenatal bipolar disorder (no) & 99.9 & 188156 & 98.9 & 881 & 109.2 & $<0.001$ \\
\hline & 0.1 & 141 & 1.1 & 10 & & \\
\hline Prenatal cocaine disorder (no) & 100.0 & 188292 & 99.3 & 885 & 575.7 & $<0.001$ \\
\hline & 0.0 & 5 & 0.7 & 6 & & \\
\hline Prenatal depressive disorder (no) & 99.2 & 186836 & 89.7 & 799 & 981.6 & $<0.001$ \\
\hline & 0.8 & 1461 & 10.3 & 92 & & \\
\hline Prenatal opioids disorder (no) & 99.7 & 187729 & 90.0 & 802 & 2376.8 & $<0.001$ \\
\hline & 0.3 & 568 & 10.0 & 89 & & \\
\hline Prenatal polydrug disorder (no) & 100.0 & 188231 & 97.5 & 869 & 1078.3 & $<0.001$ \\
\hline & 0.0 & 66 & 2.5 & 22 & & \\
\hline Prenatal alcohol disorder (no) & 99.9 & 188170 & 94.5 & 842 & 2757.2 & $<0.001$ \\
\hline & 0.1 & 127 & 5.5 & 49 & & \\
\hline Prenatal sedative disorder (no) & 100.0 & 188256 & 97.6 & 870 & 1405.6 & $<0.001$ \\
\hline & 0.0 & 41 & 2.4 & 21 & & \\
\hline Prenatal stimulant use disorder (no) & 99.9 & 188177 & 90.0 & 802 & 7826.6 & $<0.001$ \\
\hline & 0.1 & 120 & 10.0 & 89 & & \\
\hline Prenatal tobacco use disorder (no) & 99.9 & 188160 & 97.8 & 871 & 478.7 & $<0.001$ \\
\hline & 0.07 & 137 & 2.2 & 20 & & \\
\hline School type (private) & 10.4 & 19571 & 1.6 & 14 & 254.3 & $<0.001$ \\
\hline Catholic & 22.1 & 41706 & 5.9 & 53 & & \\
\hline Government & 67.5 & 127020 & 92.4 & 824 & & \\
\hline LBOTE (no) & 71.1 & 132400 & 95.0 & 843 & 245.6 & $<0.001$ \\
\hline yes & 28.9 & 53811 & 5.0 & & & \\
\hline
\end{tabular}




\begin{tabular}{|ccccccc} 
SEIFA quartile (1st) & 24.5 & 46159 & 35.8 & 319 & 207.7 & $<0.001$ \\
2nd & 20.4 & 38416 & 28.8 & 257 & & \\
3rd & 24.0 & 45249 & 25.6 & 228 & & \\
4th (wealthiest) & 31.1 & 58473 & 10.0 & 87 & & \\
Maternal age & & & & & & \\
$12-17$ & 0.9 & 1638 & 6.4 & 57 & 748.6 & $<0.001$ \\
$18-24$ & 19.8 & 36796 & 45.4 & 403 & & \\
$25-29$ & 30.6 & 56896 & 28.5 & 253 & & \\
$30-34$ & 33.2 & 61705 & 12.2 & 108 & & \\
$35+$ & 15.6 & 29089 & 7.4 & 66 & & \\
Previous births & & & & & & \\
\hline 0 & 42.1 & 78360 & 38.2 & 339 & 87.0 & \\
1 & 34.6 & 64436 & 25.6 & 227 & & \\
$2+$ & 23.3 & 43328 & 36.2 & 321 & & \\
\hline
\end{tabular}

This article is protected by copyright. All rights reserved. 
Table 2: Associations between prenatal CUD and NAPLAN results

\begin{tabular}{lccccc}
\hline \multicolumn{1}{c}{ Reading } & Spelling & Grammar & Writing & Numeracy \\
& OR $(95 \% \mathrm{Cl})$ & OR $(95 \% \mathrm{Cl})$ & OR $(95 \% \mathrm{Cl})$ & OR $(95 \% \mathrm{Cl})$ & OR $(95 \% \mathrm{Cl})$ \\
\hline Prenatal CUD (unmatched) & $3.42(2.94,3.99)$ & $4.17(3.55,4.91)$ & $3.75(3.28,4.28)$ & $4.04(3.55,4.60)$ & $3.55(2.99,4.22)$ \\
Prenatal CUD (matched) & $1.11(0.89,1.37)$ & $1.35(1.07,1.70)$ & $1.40(1.17,1.68)$ & $1.31(1.09,1.57)$ & $1.25(0.98,1.59)$ \\
\hline Note: 'unmatched' refers to the full sample (unadjusted). & &
\end{tabular}
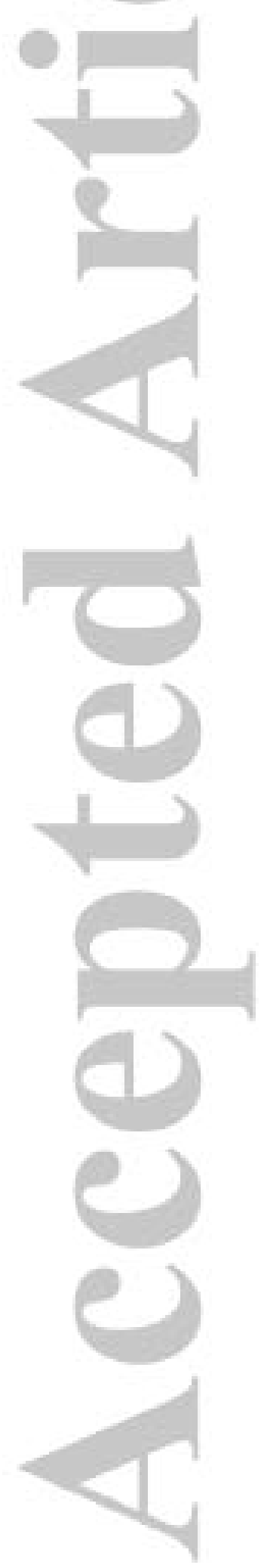

This article is protected by copyright. All rights reserved. 
Table 3: Associations between prenatal AUD and OUD with NAPLAN results

\begin{tabular}{lccccc}
\hline & $\begin{array}{c}\text { Reading } \\
\text { OR }(95 \% \mathrm{Cl})\end{array}$ & $\begin{array}{c}\text { Spelling } \\
\mathrm{OR}(95 \% \mathrm{Cl})\end{array}$ & $\begin{array}{c}\text { Grammar } \\
\mathrm{OR}(95 \% \mathrm{Cl})\end{array}$ & $\begin{array}{c}\text { Writing } \\
\mathrm{OR}(95 \% \mathrm{Cl})\end{array}$ & $\begin{array}{c}\text { Numeracy } \\
\mathrm{OR}(95 \% \mathrm{Cl})\end{array}$ \\
\hline Prenatal AUD (unmatched) & $4.53(3.34,6.12)$ & $4.12(2.92,5.82)$ & $3.47(2.54,4.72)$ & $4.45(3.39,5.84)$ & $5.16(3.69,7.19)$ \\
Prenatal AUD (matched) & $1.39(0.87,2.22)$ & $1.02(0.59,1.77)$ & $1.43(0.90,2.29)$ & $1.14(0.74,1.75)$ & $2.43(1.45,4.10)$ \\
Prenatal OUD (unmatched) & $3.87(3.28,4.57)$ & $4.43(3.68,5.32)$ & $3.93(3.38,4.59)$ & $4.01(3.46,4.66)$ & $3.88(3.23,4.67)$ \\
Prenatal OUD (matched) & $1.25(0.94,1.67)$ & $1.74(1.32,2.28)$ & $1.39(1.10,1.76)$ & $1.39(1.11,1.73)$ & $1.26(0.94,1.69)$ \\
\hline
\end{tabular}

Note: 'unmatched' refers to the full sample (unadjusted).

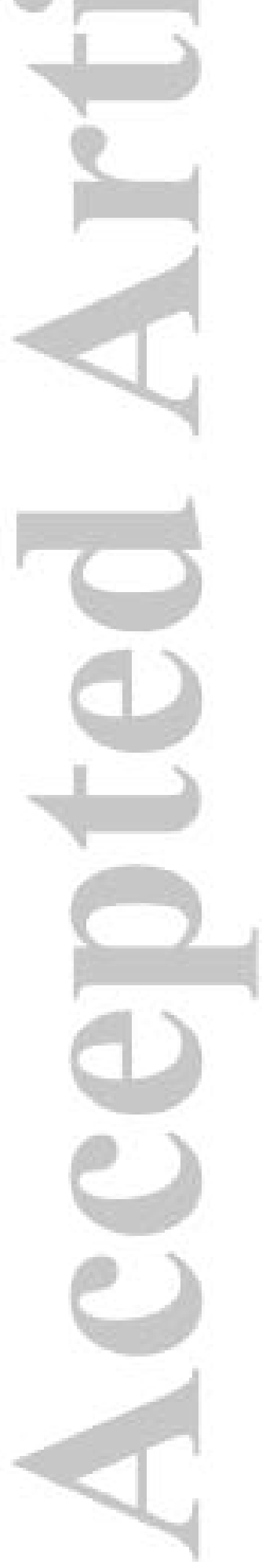

This article is protected by copyright. All rights reserved. 


\section{References}

1. Hall W, Lynskey M. Assessing the public health impacts of legalizing recreational cannabis use: the US experience. World psychiatry. 2020;19(2):179-86.

2. Volkow ND, Han B, Compton WM, McCance-Katz EF. Self-reported medical and nonmedical cannabis use among pregnant women in the United States. Jama. 2019;322(2):167-9.

3. Grzeskowiak LE, Grieger JA, Andraweera P, Knight EJ, Leemaqz S, Poston L, et al. The deleterious effects of cannabis during pregnancy on neonatal outcomes. Medical Journal of Australia. 2020.

4. Corsi DJ, Walsh L, Weiss D, Hsu H, El-Chaar D, Hawken S, et al. Association between selfreported prenatal cannabis use and maternal, perinatal, and neonatal outcomes. Jama. 2019;322(2):145-52.

5. Higuera-Matas A, Ucha M, Ambrosio E. Long-term consequences of perinatal and adolescent cannabinoid exposure on neural and psychological processes. Neuroscience \& Biobehavioral Reviews. 2015;55:119-46.

6. El Marroun H, Tiemeier H, Jaddoe VW, Hofman A, Mackenbach JP, Steegers EA, et al. Demographic, emotional and social determinants of cannabis use in early pregnancy: the Generation R study. Drug and alcohol dependence. 2008;98(3):218-26.

7. Fried PA, Watkinson B. Visuoperceptual functioning differs in 9-to 12-year olds prenatally exposed to cigarettes and marihuana. Neurotoxicology and teratology. 2000;22(1):11-20.

8. Goldschmidt L, Richardson GA, Willford J, Day NL. Prenatal marijuana exposure and intelligence test performance at age 6. Journal of the American Academy of Child \& Adolescent Psychiatry. 2008;47(3):254-63.

9. Huizink A. Prenatal cannabis exposure and infant outcomes: overview of studies. Progress in Neuro-Psychopharmacology and Biological Psychiatry. 2014;52:45-52.

10. Mereu G, Fà M, Ferraro L, Cagiano R, Antonelli T, Tattoli M, et al. Prenatal exposure to a cannabinoid agonist produces memory deficits linked to dysfunction in hippocampal long-term potentiation and glutamate release. Proceedings of the National Academy of Sciences.

2003;100(8):4915-20.

11. Campolongo P, Trezza V, Cassano T, Gaetani S, Morgese MG, Ubaldi M, et al. PRECLINICAL STUDY: Perinatal exposure to delta-9-tetrahydrocannabinol causes enduring cognitive deficits associated with alteration of cortical gene expression and neurotransmission in rats. Addiction biology. 2007;12(3-4):485-95.

12. O'Shea M, McGregor IS, Mallet PE. Repeated cannabinoid exposure during perinatal, adolescent or early adult ages produces similar longlasting deficits in object recognition and reduced social interaction in rats. Journal of psychopharmacology. 2006;20(5):611-21.

13. Scheyer AF, Melis M, Trezza V, Manzoni OJ. Consequences of perinatal cannabis exposure. Trends in Neurosciences. 2019;42(12):871-84.

14. Daniel JZ, Hickman M, Macleod J, Wiles N, LINGFORD-HUGHES A, Farrell M, et al. Is socioeconomic status in early life associated with drug use? A systematic review of the evidence. Drug and alcohol review. 2009;28(2):142-53.

15. Redonnet B, Chollet A, Fombonne E, Bowes L, Melchior M. Tobacco, alcohol, cannabis and other illegal drug use among young adults: the socioeconomic context. Drug and alcohol dependence. 2012;121(3):231-9.

16. Agrawal A, Neale MC, Prescott CA, Kendler KS. Cannabis and other illicit drugs: comorbid use and abuse/dependence in males and females. Behavior genetics. 2004;34(3):217-28.

17. Badiani A, Boden JM, De Pirro S, Fergusson DM, Horwood L, Harold GT. Tobacco smoking and cannabis use in a longitudinal birth cohort: evidence of reciprocal causal relationships. Drug and alcohol dependence. 2015;150:69-76.

This article is protected by copyright. All rights reserved. 
18. Degenhardt L, Hall W, Lynskey M. The relationship between cannabis use, depression and anxiety among Australian adults: findings from the National Survey of Mental Health and Well-Being. Social psychiatry and psychiatric epidemiology. 2001;36(5):219-27.

19. Salom CL, Betts KS, Williams GM, Najman JM, Alati R. Predictors of comorbid polysubstance use and mental health disorders in young adults-a latent class analysis. Addiction. 2016;111(1):15664. 20. Coffey C, Lynskey M, Wolfe R, Patton G. Initiation and progression of cannabis use in a population-based Australian adolescent longitudinal study. Addiction. 2000;95(11):1679-90.

21. Linkage CfHR. Master Linkage Key (MLK) 2020 [Available from:

https://www.cherel.org.au/master-linkage-key.

22. Authority ACAaR. NAPLAN 2020 [Available from: https://www.nap.edu.au/results-andreports/how-to-interpret.

23. Carey. V, J. Generalized Estimation Equation Solver. 2015.

24. Stuart EA, King G, Imai K, Ho D. Matchlt: nonparametric preprocessing for parametric causal inference. Journal of statistical software. 2011.

25. Lumley. T. Analysis of Complex Survey Samples. 2017.

26. Obstetricians ACo, Gynecologists, Women CoHCfU. Committee Opinion No. 496: at-risk drinking and alcohol dependence: obstetric and gynecologic implications. Obstetrics and gynecology. 2011;118(2 Pt 1):383.

27. Silverstein M, Howell EA, Zuckerman B. Cannabis use in pregnancy: a tale of 2 concerns. Jama. 2019;322(2):121-2.

28. Hurd Y, Wang X, Anderson V, Beck O, Minkoff H, Dow-Edwards D. Marijuana impairs growth in mid-gestation fetuses. Neurotoxicology and teratology. 2005;27(2):221-9.

29. Paul SE, Hatoum AS, Fine JD, Johnson EC, Hansen I, Karcher NR, et al. Associations Between Prenatal Cannabis Exposure and Childhood Outcomes: Results From the ABCD Study. JAMA psychiatry.

30. Chan GC, Leung J, Quinn C, Weier M, Hall W. Socio-economic differentials in cannabis use trends in Australia. Addiction. 2018;113(3):454-61.

31. Passey ME, Sanson-Fisher RW, D’Este CA, Stirling JM. Tobacco, alcohol and cannabis use during pregnancy: clustering of risks. Drug and Alcohol Dependence. 2014;134:44-50.

This article is protected by copyright. All rights reserved. 